\title{
Neonatal Sepsis: Mortality and Morbidity in Neonatal Sepsis due to Multidrug-Resistant (MDR) Organisms: Part 1
}

\author{
Chand Wattal $^{1}$ - Neelam Kler ${ }^{2}$. J. K. Oberoi ${ }^{1}$ - Anurag Fursule ${ }^{2} \cdot$ Anup Kumar $^{2} \cdot$ Anup Thakur $^{2}$
}

Received: 21 August 2019 / Accepted: 22 October 2019 / Published online: 11 December 2019

(C) The Author(s) 2019

\begin{abstract}
The major causes of emergence of multidrug-resistant organisms (MDRO) in neonatal sepsis include empiric antibiotic prescriptions, unregulated use of over-the-counter drugs, high incidence of healthcare associated infections (HAI), lack of awareness about antibiotic stewardship program and under staffing of neonatal intensive care units. In general, mortality due to MDRO sepsis is significantly higher as compared to non MDRO sepsis. Reported morbidities include prolonged use of total parenteral nutrition, need for central venous catheter, invasive ventilation, increased duration of hospital stay and neurologic sequelae.
\end{abstract}

Keywords Neonatal sepsis $\cdot$ MDR $\cdot$ Mortality $\cdot$ Morbidity $\cdot$ Financial burden

\section{Introduction}

Neonatal sepsis is a clinical syndrome characterized by systemic signs and symptoms of infection and is accompanied by bacteremia in the first month of life [1]. Early-onset sepsis (EOS) is defined as sepsis occurring in the first $72 \mathrm{~h}$ of life and that occurring beyond $72 \mathrm{~h}$ is defined as late-onset sepsis (LOS) [2]. As per World Health Organization (WHO), neonatal sepsis is the third most frequent etiology of neonatal mortality [3]. In the year 2013, a systematic analysis of global, national and regional causes of child mortality found neonatal sepsis to be the leading cause of neonatal deaths in India [4, 5]. The National Neonatal Perinatal Database network (NNPD, 2002-03) comprising of 18 tertiary care neonatal units across India reported sepsis (septicemia/meningitis) as the commonest cause of neonatal mortality, causing $23.4 \%$ of all neonatal deaths [6].

The pattern of the bacterial pathogens responsible for neonatal sepsis has changed temporally and geographically. There is a difference in the causative organisms for neonatal sepsis between the developed and developing countries [2,7]. As per NNPD, Klebsiella pneumoniae and Staphylococcus aureus

Chand Wattal

chandwattal@gmail.com

1 Department of Clinical Microbiology \& Immunology, Sir Ganga Ram Hospital, New Delhi, India

2 Department of Neonatology, Sir Ganga Ram Hospital, New Delhi, India are the commonest causative organisms for EOS and LOS in India [6]. On the contrary, data from developed countries shows that gram-positive organisms are the predominant causes of EOS as well as LOS [2, 8].

The ability of bacteria to resist or to become tolerant to several structurally and functionally distinct drugs simultaneously is known as multidrug resistance [9]. Simpler definitions quote "multidrug-resistant organisms (MDROs) are labelled as such because of their in-vitro resistance to more than one antimicrobial agent". On the other hand, definitions vary as per specific organism [10]. It is estimated that in India, 56,524 neonatal deaths each year are attributed to isolates resistant to first-line antibiotics [11].

\section{Multidrug Resistance: Global Picture}

A recent point prevalence study - Antibiotic Resistance and Prescribing in European Children (ARPEC) was conducted in 226 hospitals (41 countries) which also included NICU data from our institute (Sir Ganga Ram Hospital, New Delhi). This survey showed that most commonly used regimen for neonatal sepsis was combination of ampicillin/amoxicillin/benzyl penicillin and aminoglycoside. It further reported that $40 \%$ pathogens isolated were resistant to first-line antibiotics prescribed by WHO [12]. Though this survey had paucity of data from low- and middle-income countries (LMICs), it provided important insights on emergence of antibiotic resistance. The 
resistance to first-line antibiotics in different WHO regions and is given in Table 1 [13].

Estimates of MDRO burden have also been reported from other countries. In a systematic review from five countries of South Asia (India, Pakistan, Sri Lanka, Bangladesh and Nepal) comprising of 109 studies, a high proportion of MDRO was reported. The pooled estimated data from hospital and community showed that Klebsiella pneumoniae, E. coli and Acinetobacter baumannii were multidrug resistant in $70.7 \%, 54 \%, 78.7 \%$ of isolates respectively [15]. A retrospective single centre study from Jordan evaluated 4 y data of 68 episodes of culture positive neonatal sepsis. Gram negative organisms were the commonest and $69 \%$ of these were multidrug resistant [16]. In another cohort study from Taiwan, conducted over 8 y, 1106 episodes of culture positive sepsis were reported. Of these, one-third were caused by gram negative bacilli and $70(18.6 \%)$ were multidrug resistant [17]. A meta-analysis of 71 studies reported from China showed that $50 \%$ of gram negative organisms were resistant to thirdgeneration cephalosporins [18].

\section{Multidrug Resistance: Scenario in India}

A study of microbiological profile of $E$. coli from three NICUs in India which included 67 neonates with $E$. coli sepsis reported that majority of the isolates were resistant to cefotaxime ( $87 \%)$, ciprofloxacin $(70 \%)$ and trimethoprim/ sulfamethoxazole (76\%). Phenotypic tests demonstrated that $87 \%$ of isolates were extended $\beta$ lactamases (ESBL) producers and $6 \%$ were metallo $\beta$ lactamases (MBL) producers. Nine isolates which were meropenem resistant possessed New Delhi metallo $\beta$ lactamases-1 (NDM-1) [19]. In another study from Kolkata, carbapenem resistance in neonatal sepsis was evaluated over 5 y. Fourteen percent of isolates were carbapenemese resistant and in all of them, NDM-1 was identified [20]. Similarly, Chatterjee et al. observed that $56 \%$ of Acinetobacter baumannii isolated in blood culture were carbapenem resistant and 22\% had NDM-1 [21].

A recent study, Delhi Neonatal Infection Study (DeNIS) reported clinical and microbiological data from three large tertiary care NICUs on 1005 culture positive cases [22].

Table 1 Resistance to first-line antibiotics in different WHO regions

\begin{tabular}{lll}
\hline $\begin{array}{l}\text { Region as defined } \\
\text { by WHO [14] }\end{array}$ & $\begin{array}{l}\text { Resistance to } \\
\text { Ampicillin (\%) }\end{array}$ & $\begin{array}{l}\text { Resistance to } \\
\text { Gentamicin (\%) }\end{array}$ \\
\hline SEARO & 97 & 83 \\
AFRO & 93 & 43 \\
EURO & 64 & 13
\end{tabular}

SEARO WHO South East Asian region; AFRO WHO Africa region; EURO WHO Europe region
Two-third of isolates were gram-negative. Commonest organisms isolated were Acinetobacter baumannii (22\%) followed by Klebsiella pneumoniae (17\%) and Escherichia coli (14\%). There were high rates of multidrug resistance (resistance to any three of five antibiotic classes) in Acinetobacter baumannii (82\%), Klebsiella pneumoniae (54\%), and Escherichia coli (38\%) isolates. In another cohort study from Delhi, multi-drug resistance rates in Klebsiella pneumoniae, A. baumannii, E. coli, E. cloacae were reported to be $65.4 \%$, $71.4 \%, 78.7 \%$ and 66.0 respectively [23].

In a recent randomized controlled trial conducted at Sir Ganga Ram Hospital, New Delhi, out of 50 infants with gram negative sepsis, $54 \%$ isolates were MDROs. Fifty eight percent of Klebsiella pneumoniae, $62.5 \%$ of Burkholderia cepacia and $66.6 \%$ of Acinetobacter baumanii were multidrug resistant.

\section{Risk Factors for Development of Antimicrobial Resistance}

Global consumption of antibiotics has risen by $36 \%$ from 2000 to 2010. BRICS countries (Brazil, Russia, India, China, and South Africa) contributed to $75 \%$ of this increase inspite of overall representation of only $40 \%$ of the world's population [24]. In India, poor enforcement of regulations of over-thecounter sales of antibiotics, its low cost and an increase in consumption due to economic growth and prosperity are important factors implicated in rise of antibiotic resistance [24].

The major factors responsible for emergence and propagation of antimicrobial resistance (AMR) are overuse and empiric use of antibiotics, poor infection control in clinics and hospitals [25], lack of knowledge regarding organisms and its inherent antibiogram and rampant use of unreasonable fixed drug combinations [25-28]. Understaffing of NICUs are a major concern in resource limited developing countries [29]. Recent extensive use of antibiotics in agriculture has compounded the already existing problem of AMR. Antimicrobial growth promotors play a crucial role in this industry since they improve feed conversion, animal growth and reduce morbidity and mortality due to clinical and subclinical diseases [30].

\section{Outcomes in MDRO Sepsis}

\section{Mortality}

Various studies conducted globally have reported higher case fatality rates (CFR) due to MDRO sepsis. A study from Jordan reported that sepsis due to MDROs was associated with significantly higher mortality rate as compared to non-MDRO sepsis $(60 \%$ vs. 13\%) [16]. Similarly, another study from Taiwan reported mortality rate of $26.3 \%$ due to MDR Acinetobacter baumanni [17]. 
From India, the DeNIS study reported that the population attributable risk of mortality was $15.7 \%$ in culture-positive sepsis by MDRO vs. $12.0 \%$ in culture-positive sepsis by nonMDRO [22]. Another study from Delhi reported CFR of culture-positive and culture negative sepsis to be $23.0 \%$ and $6.8 \%$ respectively. The CFR among MDRO was higher than sensitive isolates. Among the neonates with MDR sepsis, only half survived [23]. In a recent data (2018-2019) from Sir Ganga Ram Hospital, New Delhi 64 culture positive extramural neonates cases had high mortality in MDRO as compared with nonMDRO [34.4\% vs. $8 \%, p=0.028, \mathrm{OR}=6.02$ (C.I; $1.1-30.3)]$.

\section{Morbidities}

In a study done in Taiwan, 376 episodes of gram negative bacteremia (GNB) were analysed. Underlying neurologic sequelae ( $22.9 \%$ vs. $13.4 \%)$, renal disease $(12.9 \%$ vs. $1.3 \%$ ), previous episode of bacteremia (35.7\% vs. $23.5 \%)$, use of total parenteral nutrition ( $80 \%$ vs. $67.6 \%$ ), use of central venous catheter $(87.1 \%$ vs. $73.2 \%)$ were significantly high in MDR GNB as compared to non-MDR GNB cohort [31].

\section{Financial Implications}

Though limited data exists on cost analysis, however, data from our institute (2010) from 59 episodes of culture proven sepsis (20 multidrug resistant isolates) showed prolonged duration of hospital stay in MDRO sepsis (27.6 d) vs. non-MDRO sepsis (20.7 d). The mean cost of therapy for MDRO sepsis was INR $4,99,840$ vs. INR 180,592 for non-MDRO sepsis. On subgroup analysis of infants $<1000 \mathrm{~g}$ birth weight, the mean cost of therapy in MDRO sepsis vs. non-MDRO sepsis showed further difference (INR 7,34,798 vs. 2,50,558) [32].

A study from USA reported escalation of cost of therapy due to ESBL Klebsiella outbreak in NICU to be 3,41,751 dollars, with major fraction of increase in cost due to health care provider time engaged in patient care [33]. Gandra et al. estimated the economic burden due to antibiotic resistance. Despite methodological limitation and difference in ways of estimation of disease burden, excess cost attributable due to infection caused by resistant organism $v s$. sensitive organism was much higher (Table 2) [34].

\section{Tackling Antimicrobial Resistance}

Adherence to optimum hand hygiene practice is a cardinal step to prevent infection with MDRO. Antimicrobial stewardship programs help to optimize clinical outcomes, reducing untoward consequences of antibiotic use like toxicity and the emergence of resistance [35]. In a recent study in a single NICU, Jinka et al. found that implementation of antimicrobial policy based on microbiology information for neonatal sepsis
Table 2 Surplus expenditure in MDRO

\begin{tabular}{|c|c|c|}
\hline Resistant Organism & Control & $\begin{array}{l}\text { Range of Excess } \\
\text { Cost }\end{array}$ \\
\hline MRSA & MSSA & $\$ 695-\$ 29,030$ \\
\hline $\begin{array}{l}\text { Vancomycin resistant } \\
\text { Enterococcus }\end{array}$ & $\begin{array}{l}\text { Vancomycin susceptible } \\
\text { Enterococcus }\end{array}$ & $\$ 16,711-\$ 60,988$ \\
\hline $\begin{array}{l}\text { Resistant } P \text { seudomonas } \\
\quad \text { aeruginosa }\end{array}$ & Susceptible $P$. aeruginosa & $\$ 627-\$ 45,256$ \\
\hline $\begin{array}{l}\text { Resistant Acinetobacter } \\
\quad \text { baumannii }\end{array}$ & Susceptible A.baumannii & $\$ 5336-\$ 126,856$ \\
\hline Multiple organisms & Susceptible & $\$ 9372-\$ 18,990$ \\
\hline $\begin{array}{l}\text { ESBL producing } \\
\text { Enterobacteriaceae }\end{array}$ & $\begin{array}{c}\text { Non-ESBL producing } \\
\text { Enterobacteriaceae }\end{array}$ & $\$ 3658-\$ 4892$ \\
\hline
\end{tabular}

ESBL Extended $\beta$ lactamases; MRSA Methicillin resistant Staphylococcus aureus; MSSA Methicillin sensitive Staphylococcus aureus

resulted in increased use of first-line agents and reduced use of third-generation cephalosporins without effecting patient outcomes [36].

Regulation for over-the-counter drugs sale laid down by Central Drugs Standard Control Organization (CDSCO) has been a step to tackle AMR in India [37]. The Indian National Action Plan on AMR (2017-21) aims to gradually eliminate animal use of critically important antibiotics used on humans [38]. Other measures include optimizing antenatal care, intrapartum care, postnatal care and reducing use in suspected viral infections [15].

\section{Conclusions}

Resistance is surging rapidly in LMIC countries. Infection with MDRO leads to higher mortality and morbidity in neonates. The economic burden is exponentially increased in MDRO sepsis. Risk factors for spread of MDRO include indiscriminate use of antibiotics, lack of hand hygiene, poor antibiotic stewardship and other socioeconomic factors including poor sanitation, lack of nurse patient ratio and overcrowding. Tackling spread of MDRO is an emergency and recommendations of $\mathrm{WHO} / \mathrm{CDC}$ should be implemented with utmost sincerity in units.

\section{Compliance with Ethical Standards}

Conflict of Interest None.

Open Access This article is distributed under the terms of the Creative Commons Attribution 4.0 International License (http:// creativecommons.org/licenses/by/4.0/), which permits unrestricted use, distribution, and reproduction in any medium, provided you give 
appropriate credit to the original author(s) and the source, provide a link to the Creative Commons license, and indicate if changes were made.

\section{References}

1. Klein JO. Bacteriology of neonatal sepsis. Pediatr Infect Dis J. 1990;9:778.

2. Stoll BJ, Hansen NI, Sanchez PJ, et al. Early onset neonatal sepsis: the burden of group B streptococcal and E. coli disease continues. Pediatrics. 2011;127:817-26.

3. Liu L, Oza S, Hogan D, et al. Global, regional, and national causes of under-5 mortality in 2000-15: an updated systematic analysis with implications for the sustainable development goals. Lancet. 2016;388:3027-35.

4. Liu L, Johnson HL, Cousens S, et al. Global, regional, and national causes of child mortality: an updated systematic analysis for 2010 with time trends since 2000. Lancet. 2012;379:2151-61.

5. Liu L, Oza S, Hogan D, et al. Global, regional, and national causes of child mortality in 2000-13, with projections to inform post-2015 priorities: an updated systematic analysis. Lancet. 2015;385:430-40.

6. Deorari A, Agrawal R, Paul VK, Agrawal R, Upadhayay A, Chawla D GG. National Neonatal- Perinatal Database. NNPD Nodal Center AIIMS Delhi. New Delhi; 2005. Available at: https://www.newbornwhocc.org/pdf/nnpd_report_2002-03.PDF. Accessed 10 August 2019.

7. Zaidi A, Thaver D, Ali S, Khan T. Pathogens associated with sepsis in newborns and young infants in developing countries. Pediatr Infect Dis J. 2009;28:S10-8.

8. Stoll BJ, Hansen N, Fanaroff AA, et al. Late-onset sepsis in very low birth weight neonates: the experience of the NICHD neonatal research network. Pediatrics. 2002;110:285-91.

9. Drug Resistance, Multiple, Bacterial - MeSH - NCBI [Internet]. Available at: https://www.ncbi.nlm.nih.gov/mesh/68024901. Accessed 9 August 2019.

10. Magiorakos AP, Srinivasan A, Carey RB, et al. Multidrugresistant, extensively drug-resistant and pandrug-resistant bacteria: an international expert proposal for interim standard definitions for acquired resistance. Clin Microbiol Infect. 2012;18:268-81.

11. Laxminarayan R, Matsoso P, Pant S, et al. Access to effective antimicrobials: a worldwide challenge. Lancet. 2016;387:168-75.

12. Versporten A, Bielicki J, Drapier N, Sharland M, Goossens H. ARPEC Project Group. The worldwide antibiotic resistance and prescribing in European children (ARPEC) point prevalence survey: developing hospital-quality indicators of antibiotic prescribing for children. J Antimicrob Chemother. 2016;71:1106-17.

13. WHO. GARDP Program Strategy Neonatal Sepsis: NeoAMR. 2016.

14. WHO Medicines Strategy: Framework for Action in Essential Drugs and Medicines Policy - 2000 - 2003: Abbreviations, Acronyms \&amp; WHO Regions [Internet]. Available at: http:// apps.who.int/medicinedocs/en/d/Jwhozip16e/2.html. Accessed 6 Aug 2019.

15. Chaurasia S, Sivanandan S, Agarwal R, Ellis S, Sharland M, Sankar MJ. Neonatal sepsis in South Asia: huge burden and spiralling antimicrobial resistance. BMJ. 2019;364:k5314.

16. Yusef D, Shalakhti T, Awad S, Algharaibeh H, Khasawneh W. Clinical characteristics and epidemiology of sepsis in the neonatal intensive care unit in the era of multi-drug resistant organisms: a retrospective review. Pediatr Neonatol. 2018;59: $35-41$.

17. Wei HM, Hsu YL, Lin HC, et al. Multidrug-resistant Acinetobacter baumannii infection among neonates in a neonatal intensive care unit at a medical center in Central Taiwan. J Microbiol Immunol Infect. 2015;48:531-9.
18. Li JY, Chen SQ, Yan YY, et al. Identification and antimicrobial resistance of pathogens in neonatal septicemia in China - a metaanalysis. Int J Infect Dis. 2018;71:89-93.

19. Roy S, Datta S, Das P, et al. Insight into neonatal septicaemic Escherichia coli from India with respect to phylogroups, serotypes, virulence, extended-spectrum- $\beta$-lactamases and association of ST131 clonal group. Epidemiol Infect. 2015;143:3266-76.

20. Datta S, Roy S, Chatterjee S, et al. A five-year experience of carbapenem resistance in enterobacteriaceae causing neonatal septicaemia: predominance of NDM-1. PLoS One. 2014;9: e112101.

21. Chatterjee S, Datta S, Roy S, et al. Carbapenem resistance in Acinetobacter baumannii and other Acinetobacter spp. causing neonatal sepsis: focus on NDM-1 and its linkage to ISAba125. Front Microbiol. 2016;7:1126.

22. Investigators of the Delhi Neonatal Infection Study (DeNIS) Collaboration. Characterisation and antimicrobial resistance of sepsis pathogens in neonates born in tertiary care centres in Delhi, India: a cohort study. Lancet Glob Heal. 2016;4: e752-60.

23. Jajoo M, Manchanda V, Chaurasia S, et al. Alarming rates of antimicrobial resistance and fungal sepsis in outborn neonates in North India. PLoS One. 2018;13:e0180705.

24. Van Boeckel TP, Gandra S, Ashok A, et al. Global antibiotic consumption 2000 to 2010: an analysis of national pharmaceutical sales data. Lancet Infect Dis. 2014;14:742-50.

25. WHO. Antimicrobial Resistance [Internet]. Available at: https:// www.who.int/news-room/fact-sheets/detail/antimicrobialresistance. Accessed 30 July 2019.

26. Smith RD, Coast J. Antimicrobial resistance: a global response. Bull World Health Org. 2002;80:126-33.

27. WHO. 10 Facts on Antimicrobial Resistance [Internet]. WHO. World Health Organization; 2017. Available at: https://www.who. int/features/factfiles/antimicrobial_resistance/en/. Accessed 31 July 2019.

28. Goswami N, Gandhi A, Patel P, Dikshit R. An evaluation of knowledge, attitude and practices about prescribing fixed dose combinations among resident doctors. Perspect Clin Res. 2013;4:130-5.

29. Stapleton PJ, Murphy M, McCallion N, Brennan M, Cunney R, Drew RJ. Outbreaks of extended spectrum beta-lactamaseproducing Enterobacteriaceae in neonatal intensive care units: a systematic review. Arch Dis Child Fetal Neonatal Ed. 2016;101: F72-8.

30. Butaye P, Devriese LA, Haesebrouck F. Antimicrobial growth promoters used in animal feed: effects of less well known antibiotics on gram-positive bacteria. Clin Microbiol Rev. 2003;16:175-88.

31. Tsai MH, Chu SM, Hsu JF, et al. Risk factors and outcomes for multidrug-resistant gram-negative bacteremia in the NICU. Pediatrics. 2014;133:e322-9.

32. Oberoi JK, Wattal C. Neonatal sepsis: an outcome study at a tertiary care centre in New Delhi, India. Poster \& Oral presentation presented at "1st Global Forum on Bacterial Infections" at New Delhi on 3-5 October 2011.

33. Stone PW, Gupta A, Loughrey M, et al. Attributable costs and length of stay of an extended-spectrum beta-lactamase-producing Klebsiella pneumoniae outbreak in a neonatal intensive care unit. Infect Control Hosp Epidemiol. 2003;24:601-6.

34. Gandra S, Barter DM, Laxminarayan R. Economic burden of antibiotic resistance: how much do we really know? Clin Microbiol Infect. 2014;20:973-80.

35. Dellit TH, Owens RC, McGowan JE, et al. Infectious Diseases Society of America and the Society for Healthcare Epidemiology of America guidelines for developing an institutional program to enhance antimicrobial stewardship. Clin Infect Dis. 2007;44:15977 . 
36. Jinka DR, Gandra S, Alvarez-Uria G, Torre N, Tadepalli D, Nayakanti RR. Impact of antibiotic policy on antibiotic consumption in a neonatal intensive care unit in India. Indian Pediatr. 2017;54:739-41.

37. Press Information Bureau, Ministry of Health and Family Welfare, India. Rules for Selling of Drugs Under Schedule H1 [Internet]. 2013. Available at: http://pib.nic.in/newsite/ PrintRelease. aspx?relid=101251. Accessed 1 Aug 2019.
38. Dutta SS. India launches strategy to curb antimicrobial resistance. BMJ. 2017;357:j2049.

Publisher's Note Springer Nature remains neutral with regard to jurisdictional claims in published maps and institutional affiliations. 